\title{
Impact of Regular Exercise and Attempted Weight Loss on Quality of Life among Adults with and without Type 2 Diabetes Mellitus
}

\author{
Andrew J. Green, ${ }^{1}$ Kathleen M. Fox, ${ }^{2}$ and Susan Grandy ${ }^{3}$ \\ ${ }^{1}$ Midwestern Endocrinology, Overland Park, KS 66211, USA \\ ${ }^{2}$ Strategic Healthcare Solutions, LLC, P.O. Box 543, Monkton, MD 21111, USA \\ ${ }^{3}$ Department of Health Economics and Outcomes Research, AstraZeneca LP, Wilmington, DE 19850, USA
}

Correspondence should be addressed to Kathleen M. Fox, kathyfox@gforcecable.com

Received 21 May 2010; Accepted 1 September 2010

Academic Editor: Neil King

Copyright (C) 2011 Andrew J. Green et al. This is an open access article distributed under the Creative Commons Attribution License, which permits unrestricted use, distribution, and reproduction in any medium, provided the original work is properly cited.

Objective. To examine the association between exercising regularly and trying to lose weight, and quality of life among individuals with and without type 2 diabetes mellitus (T2DM). Methods. Respondents to the US SHIELD baseline survey reported whether they had tried to lose weight during the previous 12 months and whether they exercised regularly for $>6$ months. Respondents completed the SF-12 quality-of-life survey one year later. Differences between T2DM respondents $(n=2419)$ and respondents with no diabetes $(n=6750)$ were tested using $t$-tests and linear regression models adjusting for demographics, body mass index (BMI), and diabetes status. Results. After adjustment, exercising regularly was significantly associated with higher subsequent physical and mental component scores $(P<.001)$. After adjustment, trying to lose weight was not associated with higher physical component scores $(P=.87)$, but was associated with higher mental component scores $(P=.01)$. Conclusion. Respondents who reported exercising regularly had significantly better physical and mental quality of life, compared with respondents who did not exercise regularly. Despite exercising regularly, respondents with T2DM had significantly worse quality of life, compared with respondents without diabetes who exercised regularly.

\section{Introduction}

Diabetes mellitus is a prevalent and costly disease. Across the world, there are 285 million adults, aged 20-79 years, with diabetes [1]. This is projected to increase to 439 million people globally by 2030 [1]. In the United States, there are 23.6 million adults 20 years or older with diabetes, and approximately $90 \%$ of them have type 2 diabetes mellitus (T2DM) [2]. Approximately 24\% of the 23.6 million Americans have undiagnosed diabetes which has not come to medical attention [2]. An additional 57 million people in the US have prediabetes, increasing their risk of developing frank diabetes [2]. The increasing prevalence of T2DM is directly related to an increasing rise in the prevalence of physical inactivity and obesity, with an estimated 97 million US adults being overweight or obese [3, 4]. Approximately two-thirds of US adult men and women diagnosed with T2DM have a body mass index (BMI) of $27 \mathrm{~kg} / \mathrm{m}^{2}$ or greater [5]. National surveys have reported that $27 \%$ of US adults did not engage in any physical activity and another $28 \%$ were not regularly active [6].

With this global burden, it is important to manage and control diabetes to prevent development of complications. Regular exercise and weight management are key selfmanagement treatments for individuals with T2DM. The American Diabetes Association (ADA) Standards of Medical Care in Diabetes [7] recommend that patients with impaired glucose tolerance or a hemoglobin A1c of $5.7 \%-6.4 \%$ be referred to a program for weight loss of 5\%-10\% of body weight and an increase of at least $150 \mathrm{~min} /$ week of moderate activity to prevent or delay T2DM. For patients with T2DM, lifestyle changes, including medical nutrition therapy and exercise, are recommended to achieve and maintain glycemic control [7]. Weight loss (at least 7\% of body weight) is recommended for all overweight or obese individuals who have diabetes, to assist in achieving and maintaining 
glycemic control. The ADA recommends that individuals with diabetes be advised to perform at least $150 \mathrm{~min} /$ week of moderate-intensity aerobic physical activity (50\%-70\% of maximum heart rate) and be encouraged to perform resistance training three times per week [7].

Individuals are often counseled by their physicians and other healthcare providers regarding weight management and exercise $[8,9]$. However, the extent to which these recommendations are incorporated into daily life among individuals with T2DM and whether they impact quality of life are unknown. This study examined the association between exercising regularly and trying to lose weight and health-related quality of life (HRQoL) among individuals with and without T2DM to determine if adults who exercised regularly or attempted weight loss had better quality of life than those who did not perform these lifestyle behaviors.

\section{Methods}

The present investigation is a longitudinal analysis of the Study to Help Improve Early evaluation and management of risk factors Leading to Diabetes (SHIELD) data to assess the association between lifestyle behaviors and HRQoL. SHIELD is a 5-year, survey-based study conducted to better understand patterns of health behavior and knowledge and attitudes of people living with diabetes and those with varying levels of cardiometabolic risk.

2.1. SHIELD Survey. SHIELD included an initial screening phase to identify cases of interest in the general population (e.g., diabetes mellitus), a baseline survey to follow up identified cases with a questionnaire about health status, health knowledge and attitudes, and current behaviors and treatments, and annual follow-up surveys. A detailed description of the SHIELD methodology has been published previously $[10,11]$.

In brief, the screening survey was mailed on April 1, 2004, to a stratified random sample of 200,000 US households, representative of the US population for geographic residence, household size and income, and age of head of household [12], identified by the Taylor Nelson Sofres National Family Opinion (TNS NFO) panel (Greenwich, CT). All TNS NFO surveys were voluntary, and no special incentives were provided. A response rate of $64 \%$ was obtained.

A comprehensive baseline survey was mailed in September-October 2004 to a representative sample of individuals $(n=22,001)$ who were identified in the screening survey as having self-reported type 1 diabetes mellitus or type 2 diabetes mellitus, no diabetes, or being at risk for diabetes. Each respondent group was balanced to be representative of that segment of the population for age, gender, geographic region, household size, and income for the US population, and then a random sample from each group was selected and sent the baseline survey. A response rate of $72 \%$ was obtained for the baseline survey. In August 2005, the first annual follow-up survey was mailed to all individuals selected for the baseline survey who were still enrolled in the household panel $(n=19,613)$, and a response rate of $72 \%$ was obtained. This investigation utilized the respondents who completed the baseline survey and the first annual follow-up survey.

2.2. Study Measures. Respondents were classified as having T2DM based upon their self-report of having been told by a doctor, nurse, or other healthcare professional that they had T2DM. A comparison cohort was identified as respondents who reported no diagnosis of T2DM, type 1 diabetes, gestational diabetes, or unspecified diabetes.

In the baseline survey, respondents answered survey questions on weight management and exercise. Respondents were asked to check one of the following statements about exercise: (1) I currently exercise regularly and have done so for longer than six months, (2) I currently exercise regularly, but have only begun doing so in the last six months, (3) I currently exercise some, but not regularly, (4) I currently do not exercise, but I am thinking about starting to exercise in the next six months, and (5) I currently do not exercise, and I do not intend to start exercising in the next six months. Respondents also answered the survey question worded as, "During the last 12 months, have you tried to lose weight?", with the response options of "yes" or "no."

The MOS Short-Form-12 version 2 (SF-12) was used to assess HRQoL in the follow-up survey (one year after the lifestyle behavior questions). The SF-12, the short form of the widely used SF-36, is a brief and reliable measure of overall health status [13]. The SF-12 measures eight domains of health: physical functioning, role limitations because of physical health, body pain, general health perceptions, vitality, social functioning, role limitations because of emotional problems, and mental health. The recall period was the past four weeks. SF-12 responses were scored from 0 to 100 on the Physical Component Summary (PCS) scale and Mental Component Summary (MCS) scale. Higher scores indicate better HRQoL. To simplify comparisons with the general population, norm-based scoring was used. In norm-based scoring, scores are linearly transformed to a scale with a mean of 50 and standard deviation (SD) of ten for the general population [13].

2.3. Statistical Analysis. The proportion of respondents reporting attempted weight loss or exercise regularly was computed for respondents with and without T2DM. Separate analyses were performed for attempted weight loss and for exercising regularly. Comparisons between respondents with and without T2DM were made using chi-square tests. For exercise, respondents who reported exercising regularly for at least six months were assessed since moderate to vigorous physical activity at least three times per week is recommended by the ADA. Linear regression models were constructed to assess the association between SF-12 PCS and MCS scores and exercising regularly (yes/no) or attempted weight loss (yes/no) adjusting for age (continuous), gender (women versus men), race (white versus other races), education (college or higher versus high school diploma or less), household income ( $>\$ 35,000$ versus $\leq \$ 35,000)$, and BMI (continuous). 
TABLE 1: Characteristics of SHIELD respondents with and without type 2 diabetes mellitus reporting exercising regularly or trying to lose weight.

\begin{tabular}{|c|c|c|c|c|}
\hline \multirow[b]{2}{*}{ Characteristics } & \multicolumn{2}{|c|}{ Exercised regularly } & \multicolumn{2}{|c|}{ Tried to lose weight } \\
\hline & $\mathrm{T} 2 \mathrm{DM}(n=472)$ & $\operatorname{No} \operatorname{DM}(n=1,687)$ & $\operatorname{T} 2 \mathrm{DM}(n=1,722)$ & $\operatorname{No} \operatorname{DM}(n=4,288)$ \\
\hline Age, years, mean (SD) & $63.1(11.9)^{*}$ & $56.3(16.1)$ & $58.3(12.0)^{*}$ & $53.7(15.2)$ \\
\hline Women, \% & 50 & 54 & 64 & 65 \\
\hline White, \% & $87^{*}$ & 90 & $86^{*}$ & 90 \\
\hline Income, $\%$ with $\leq \$ 35,000 /$ year & $44^{*}$ & 31 & $52^{*}$ & 40 \\
\hline Education, \% with no more than a high school degree & $28^{*}$ & 19 & $35^{*}$ & 27 \\
\hline Baseline weight, lbs, mean (SD) & $197.4(49.6)^{*}$ & $178.0(41.4)$ & $225.4(56.6)^{*}$ & $201.7(48.0)$ \\
\hline Normal weight $\left(\mathrm{BMI}<25.0 \mathrm{~kg} / \mathrm{m}^{2}\right)$ & $17^{*}$ & 35 & $4^{*}$ & 12 \\
\hline Overweight $\quad\left(\mathrm{BMI}=25.0-29.9 \mathrm{~kg} / \mathrm{m}^{2}\right)$ & 39 & 36 & 23 & 31 \\
\hline Obese $\left(\mathrm{BMI} \geq 30 \mathrm{~kg} / \mathrm{m}^{2}\right)$ & $44^{*}$ & 29 & $73^{*}$ & 57 \\
\hline
\end{tabular}

${ }^{*} P<.05$ for comparison of T2DM versus No DM; T2DM = type 2 diabetes mellitus, $\mathrm{DM}=$ diabetes mellitus, $\mathrm{BMI}=$ body mass index

TABLE 2: SF-12 scores for respondents with and with diabetes who did and did not regularly exercise.

\begin{tabular}{lcccc}
\hline & \multicolumn{2}{c}{ Exercise regularly } & \multicolumn{2}{c}{ No regular exercise } \\
SF-12 scores & $\operatorname{T2DM}(n=472)$ & No DM $(n=1,687)$ & $\operatorname{T} 2 \mathrm{DM}(n=1,940)$ & No DM $(n=5,041)$ \\
\hline Physical component summary (PCS), mean (SD) & $44.4(11.7)^{*}$ & $49.3(10.3)^{\dagger}$ & $38.3(12.6)^{\uparrow}$ & $43.8(12.2)$ \\
Mental component summary (MCS), mean (SD) & $52.8(9.2)$ & $52.8(8.5)^{\dagger}$ & $48.3(11.5)^{\uparrow}$ & $49.4(10.6)$ \\
\hline
\end{tabular}

${ }^{*} P<.001$ for comparison of T2DM versus No DM; $P<.001$ for comparison within T2DM of exercise regularly versus no regular exercise; ${ }^{\dagger} P<.001$ for comparison within No DM of exercise regularly versus no regular exercise.

\section{Results}

There were 2,419 respondents with T2DM and 6,750 respondents without diabetes who completed the SHIELD baseline survey and first follow-up survey, and 20\% of T2DM ( $n=$ $472)$ and $25 \%$ of no diabetes $(n=1,687)$ respondents reported exercising regularly for more than six months. Many respondents reported trying to lose weight: $71 \%$ of T2DM $(n=1,722)$ and $64 \%$ of no diabetes $(n=4,288)$ respondents. For T2DM respondents, approximately $15 \%$ were currently receiving insulin and $79 \%$ were currently receiving some type of antidiabetic medication. In comparing T2DM versus no diabetes respondents separately for exercise and weight management, significantly more T2DM respondents who reported exercising regularly were older, had lower income, had less education, and had higher baseline weight and more obesity compared with those without diabetes who exercised regularly $(P<.05)$ (Table 1$)$. A significantly larger proportion of $\mathrm{T} 2 \mathrm{DM}$ respondents who reported attempted weight loss were older, had lower income, had less education, and had higher baseline weight and more obesity compared with no diabetes respondents who attempted weight loss. Among T2DM respondents, those who reported exercising regularly were older (63.1 years versus 59.2 years), had among them fewer women (50\% versus 62\%), had higher income (44\% versus $56 \%$ with income $<\$ 35,000)$, had more education (28\% versus $38 \%$ with $\leq$ high school degree), and had lower baseline weight (197 lbs versus $220 \mathrm{lbs}$ ) and less obesity (44\% versus $67 \%$ ) than T2DM respondents who did not exercise regularly $(P<.05)$. Among T2DM respondents, those who reported trying to lose weight were younger (58.3 years versus 64.0 years), had among them more women (64\% versus $48 \%$ ), and had higher baseline weight (225 lbs versus $192 \mathrm{lbs}$ ) and more obesity (73\% versus 38\%) than T2DM respondents who did not attempt to lose weight $(P<.05)$.

3.1. Exercising Regularly. Among respondents who reported exercising regularly, PCS scores were significantly lower among T2DM respondents $(P<.05)$, and MCS scores were equivalent for both groups (Table 2). Among T2DM respondents, those who exercised regularly had higher PCS and MCS scores than T2DM respondents who did not exercise regularly $(P<.001)$ (Table 2$)$. A similar pattern of higher SF-12 scores for those who exercised regularly was observed for respondents without diabetes $(P<.001)$. However, PCS and MCS scores varied by BMI category. PCS scores decreased from normal weight to morbidly obese $(P<.001)$ (Figure 1$)$, and MCS scores also decreased as weight increased, ranging from 52.6 for normal weight to 50.7 for morbidly obese $(P<.001)$. Because differences existed between T2DM and no diabetes respondents for age, race, income, education, and $\mathrm{BMI}$, multivariate regression modeling was performed.

3.2. Attempted Weight Loss. PCS and MCS scores were significantly lower among T2DM respondents, compared with no diabetes respondents $(P<.05)$ (Table 3$)$. For T2DM respondents, PCS and MCS scores were lower among those who tried to lose weight, compared with respondents who did not try to lose weight $(P<.05)$. A similar pattern of lower PCS and MCS scores for those who tried to lose weight was observed for respondents without diabetes $(P<.001)$. Both PCS (Figure 1) and MCS scores decreased as weight increased for respondents who reported trying to lose weight. 
TABLE 3: SF-12 scores for respondents with and without diabetes who did and did not try to lose weight.

\begin{tabular}{lcccc}
\hline & \multicolumn{2}{c}{ Tried to lose weight } & \multicolumn{2}{c}{ No attempt to lose weight } \\
SF-12 scores & $\mathrm{T} 2 \mathrm{DM}(n=1,722)$ & No DM $(n=4,288)$ & $\mathrm{T} 2 \mathrm{DM}(n=697)$ & No DM $(n=2,462)$ \\
\hline Physical component summary (PCS), mean (SD) & $39.1(12.7)^{*}$ & $45.0(11.8)^{\dagger}$ & $40.6(12.3)^{\uparrow}$ & $46.0(12.0)$ \\
Mental component summary (MCS), mean (SD) & $48.9(11.2)^{*}$ & $49.8(10.4)^{\dagger}$ & $50.0(11.2)^{\uparrow}$ & $51.2(9.7)$ \\
\hline
\end{tabular}

${ }^{*} P<.001$ for comparison of T2DM versus No DM; $P<.001$ for comparison within T2DM of tried to lose weight versus no attempt to lose weight; ${ }^{\dagger} P<.001$ for comparison within No DM of tried to lose weight versus no attempt to lose weight.

TABLE 4: Multivariate linear regression results for SF-12 physical and mental component summary scores.

\begin{tabular}{|c|c|c|c|c|}
\hline \multirow[b]{2}{*}{ Model variables } & \multicolumn{2}{|c|}{ SF-12 Physical Component Summary } & \multicolumn{2}{|c|}{ SF-12 Mental Component Summary } \\
\hline & Beta coefficient & $P$-value & Beta coefficient & $P$-value \\
\hline Exercise regularly for $>6$ months $($ reference $=$ no) & 2.10 & $<.0001$ & 1.43 & $<.0001$ \\
\hline Tried to lose weight $($ reference $=$ no $)$ & 0.041 & .87 & 0.59 & .013 \\
\hline Type 2 diabetes mellitus (reference $=$ no DM) & $2.41-$ & $<.0001$ & $0.52-$ & .034 \\
\hline Age, years & $0.26-$ & $<.0001$ & 0.13 & $<.0001$ \\
\hline Gender $($ reference $=$ men $)$ & $2.21-$ & $<.0001$ & $1.69-$ & $<.0001$ \\
\hline White (reference $=$ other race $)$ & $0.02-$ & .92 & $0.09-$ & .55 \\
\hline Education (reference $=\leq$ high school degree) & 0.24 & $<.0001$ & 0.15 & .022 \\
\hline Income $($ reference $=\leq \$ 35,000 /$ year $)$ & 1.52 & $<.0001$ & 1.05 & $<.0001$ \\
\hline Body mass index, $\mathrm{kg} / \mathrm{m}^{2}$ & $0.44-$ & $<.0001$ & -0.07 & $<.0001$ \\
\hline
\end{tabular}

R-square $=0.31, P<.0001$

MCS scores ranged from 49.6 for normal-weight respondents to 46.7 for morbidly obese respondents.

3.3. Multivariate Regression. Linear regression models (one for PCS and one for MCS) were done to adjust for the baseline differences in age, race, income, education, and BMI between diabetes groups. After adjusting for baseline demographics, BMI, and diabetes status (T2DM versus no DM), exercising regularly was significantly associated with higher subsequent PCS scores, indicating better physical quality of life for those who exercised regularly in both those with and without T2DM $(P<.0001)$ (Table 4). Respondents who regularly exercised had PCS scores that were at least double those of respondents who did not exercise regularly. After adjustment, trying to lose weight was not associated with higher PCS scores $(P=.87)$. Respondents with T2DM had PCS scores that were approximately half those of respondents without diabetes, after adjusting for demographics and BMI.

After adjusting for demographics, BMI, and diabetes status, regularly exercising was significantly associated with higher MCS scores, indicating better mental HRQoL $(P<$ .0001) (Table 4). After adjustment, trying to lose weight was independently associated with higher MCS scores $(P=$ $.01)$. In general, after adjusting for demographics and BMI, respondents with T2DM had lower MCS scores than respondents without diabetes $(P=.03)$.

\section{Discussion}

Respondents who reported exercising regularly had significantly better physical and mental quality of life, compared with respondents who reported not exercising regularly, after adjusting for baseline differences. Respondents who reported trying to lose weight had significantly better mental quality of life measures according to the SF-12-validated survey instrument [13]. There was no demonstrable improvement in physical quality of life, compared with respondents who did not attempt to lose weight, after adjusting for baseline differences. Respondents with T2DM who exercised regularly had worse HRQoL than respondents without diabetes who exercised regularly.

Previous investigations have demonstrated that individuals with T2DM had worse quality of life, compared with adults without diabetes or with the general population [14-18]. The present study expanded the evidence among individuals with T2DM to those who regularly exercised or tried to lose weight. Because lifestyle modifications, including exercise and weight management, are cornerstones of managing diabetes and attaining and maintaining metabolic control, it is important to understand how these behaviors impact HRQoL. This study demonstrates the increased relative physical and emotional burden of individuals with T2DM, as quality-of-life measures for the T2DM group were lower than those for respondents in the nondiabetic group, even among those who reported exercising regularly for a period of more than six months. Nevertheless, the SHIELD survey shows clear benefits of exercise, as qualityof-life measures were higher in both the T2DM and no diabetes groups who reported participating in a regular exercise regimen for this period. It is interesting to note that while attempts to lose weight did not result in improvement in physical quality-of-life measures over the survey period, the survey instrument documents psychological benefits in the form of improved mental quality of life. Both of these findings lend support to current practice and further justify recommending therapeutic lifestyle modification as a means 


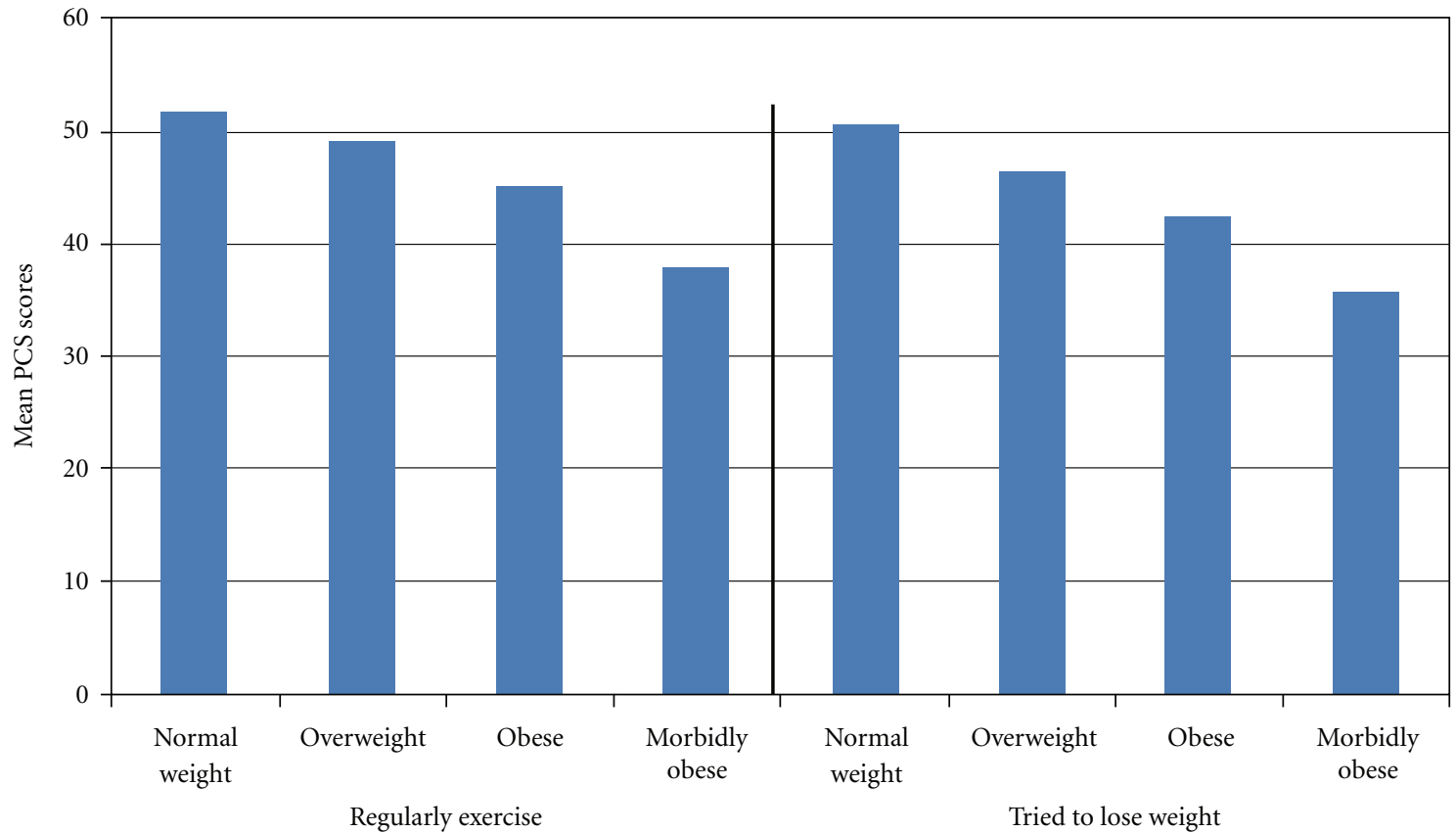

FIGURE 1: Mean Physical Component Summary scores for SHIELD respondents who reported exercising regularly or trying to lose weight by weight category. ANOVA $P<.001$; normal weight = body mass index $(\mathrm{BMI})<25.0 \mathrm{~kg} / \mathrm{m}^{2}$; overweight $=\mathrm{BMI}$ between 25.0 and $29.9 \mathrm{~kg} / \mathrm{m}^{2}$; obese $=$ BMI between 30.0 and $39.9 \mathrm{~kg} / \mathrm{m}^{2} ;$ morbidly obese $=\mathrm{BMI} \geq 40 \mathrm{~kg} / \mathrm{m}^{2}$.

of improving quality of life for individuals with T2DM over the long term, in addition to mitigating specific health risks.

The evaluation of HRQoL in this study was performed using a standardized, validated measure of overall quality of life, so that normative-based results are provided. These findings can be used for comparative analyses with other studies and with other disease conditions. However, there are limitations to the study that should be considered. The determination of T2DM was made based upon self-report rather than clinical or laboratory measures. Exercising regularly and trying to lose weight were also self-reported and not confirmed with physical expenditure measures or actual weight loss. Other studies have found that single-response items for self-reported physical activity are valid and reflect objective measures such as $\mathrm{VO}_{2}$ max, maximal exercise treadmill test, and physical activity energy expenditure [1922 . Household panels, like the SHIELD study, tend to underrepresent the very wealthy and very poor segments of the population and do not include military or institutionalized individuals. However, these limitations are true for most random sampling and clinically based methodologies. The SHIELD population is largely Caucasian which may limit the generalizability of the study findings for minorities. Selfselection bias may be present, because respondents were those who could read and comprehend the survey.

\section{Conclusions}

Respondents who reported exercising regularly for at least six months had significantly better physical and mental quality of life, compared with respondents who did not exercise regularly. Despite exercising regularly, respondents with T2DM had significantly worse quality of life, compared with respondents without diabetes who exercised regularly, further documenting the impact and burden of T2DM. Trying to lose weight had no impact on physical quality of life, but it did improve mental quality of life. These relationships were observed among individuals diagnosed with diabetes but the impact of exercise and weight management may be greater from a societal perspective if the high proportion of individuals with undiagnosed diabetes were exercising regularly or trying to lose weight. Based on the study findings, healthcare providers should continue to educate and encourage individuals with T2DM to exercise regularly and attempt to lose weight.

\section{Acknowledgments}

Members of the SHIELD Study Group are Harold Bays, MD, Louisville Metabolic and Atherosclerosis Research Center, Louisville, KY; Debbra D. Bazata, RD, CDE, St. Luke's Primary Care South, Overland Park, KS; James R. Gavin III, MD, PhD, Emory University School of Medicine, Atlanta, GA; Andrew J. Green, MD, Midwestern Endocrinology, Overland Park, KS; Sandra J. Lewis, MD, Northwest Cardiovascular Institute, Portland, OR; Michael L. Reed, PhD, Vedanta Research, Chapel Hill, NC; Helena W. Rodbard, MD, Rockville, MD. Tina Fanning of Vedanta Research, Chapel Hill, NC, also contributed to this report, performing data collection and analysis. This paper was supported by AstraZeneca LP. A. J. Green is an advisory board member for AstraZeneca LP. K. M. Fox received research funds from AstraZeneca LP to conduct the study. S. Grandy is an employee and stockholder of AstraZeneca LP. 


\section{References}

[1] International Diabetes Federation, "Diabetes Atlas," 3rd edition, April 2010, http://www.diabetesatlas.org/content/ diabetes-and-impaired-glucose-tolerance.

[2] American Diabetes Association, "Diabetes statistics," April 2010, http://www.diabetes.org/diabetes-basics/diabetesstatistics.

[3] National Heart, Lung, and Blood Institute, " Clinical Guidelines on the Identification, Evaluation, and Treatment of Overweight and Obesity in Adults: the Evidence Report," NIH Publication 98-4083, NIH, 1998.

[4] World Health Organization, "Global strategy on diet, physical activity, and health," April 2010, http://www.who.int/dietphysicalactivity/publications/facts/en/.

[5] S. Z. Yanovski, "Overweight, obesity, and health risk: National Task Force on the Prevention and Treatment of Obesity," Archives of Internal Medicine, vol. 160, no. 7, pp. 898-904, 2000.

[6] A. H. Mokdad, B. A. Bowman, E. S. Ford, F. Vinicor, J. S. Marks, and J. P. Koplan, "The continuing epidemics of obesity and diabetes in the United States," Journal of the American Medical Association, vol. 286, no. 10, pp. 1195-1200, 2001.

[7] American Diabetes Association, "Standards of Medical Care in Diabetes-2010," Diabetes Care, vol. 33 supplement 1, pp. S11-S61, 2010.

[8] A. J. Green, D. D. Bazata, K. M. Fox, and S. Grandy, "Health-related behaviours of people with diabetes and those with cardiometabolic risk factors: results from SHIELD," International Journal of Clinical Practice, vol. 61, no. 11, pp. 1791-1797, 2007.

[9] D. D. Bazata, J. G. Robinson, K. M. Fox, and S. Grandy, "Affecting behavior change in individuals with diabetes. Findings from the study to help improve early evaluation and management of risk factors leading to diabetes (SHIELD)," Diabetes Educator, vol. 34, no. 6, pp. 1025-1036, 2008.

[10] H. E. Bays, R. H. Chapman, and S. Grandy, "The relationship of body mass index to diabetes mellitus, hypertension and dyslipidaemia: comparison of data from two national surveys," International Journal of Clinical Practice, vol. 61, no. 5, pp. 737-747, 2007.

[11] H. E. Bays, D. D. Bazata, N. G. Clark et al., "Prevalence of self-reported diagnosis of diabetes mellitus and associated risk factors in a national survey in the US population: SHIELD (Study to Help Improve Early evaluation and management of risk factors Leading to Diabetes)," BMC Public Health, vol. 7, article no. 277, 2007.

[12] US Census Bureau, Annual Supplement to the Current Population Survey: Census Bureau Resident Population Estimates of the United States, US Census Bureau, Washington, DC, USA, 2003.

[13] J. E. Ware, M. Kosinski, and J. E. Dewey, How to Score Version of the SF-36 Health Survey, QualityMetric, Lincoln, RI, USA, 2000.

[14] S. Grandy, R. H. Chapman, and K. M. Fox, "Quality of life and depression of people living with type 2 diabetes mellitus and those at low and high risk for type 2 diabetes: findings from the Study to Help Improve Early evaluation and management of risk factors Leading to Diabetes (SHIELD)," International Journal of Clinical Practice, vol. 62, no. 4, pp. 562-568, 2008.

[15] F. A. Luscombe, "Health-related quality of life measurement in type 2 diabetes," Value in Health, vol. 3, no. 1, pp. S15-S28, 2000 .
[16] K. Watkins and C. M. Connell, "Measurement of healthrelated QOL in diabetes mellitus," PharmacoEconomics, vol. 22, no. 17, pp. 1109-1126, 2004.

[17] F. Mo, H. Morrison, B. C. K. Choi, and L. Vardy, "Evaluation and measurement of health-related quality of life for individuals with diabetes mellitus by Health Utilities Index Mark 3 (HUI3) system," TheScientificWorldJournal, vol. 6, pp. 14121423, 2006.

[18] W. Schlotz, P. Ambery, H. E. Syddall et al., "Specific associations of insulin resistance with impaired health-related quality of life in the Hertfordshire cohort study," Quality of Life Research, vol. 16, no. 3, pp. 429-436, 2007.

[19] A. W. Jackson, J. R. Morrow Jr., H. R. Bowles, S. J. Fitzgerald, and S. N. Blair, "Construct validity evidence for singleresponse items to estimate physical activity levels in large sample studies," Research Quarterly for Exercise and Sport, vol. 78, no. 2, pp. 24-31, 2007.

[20] M. Aadahl, M. Kjær, J. H. Kristensen, B. Mollerup, and T. Jørgensen, "Self-reported physical activity compared with maximal oxygen uptake in adults," European Journal of Cardiovascular Prevention and Rehabilitation, vol. 14, no. 3, pp. 422-428, 2007.

[21] N. Orsini, R. Bellocco, M. Bottai et al., "Validity of selfreported total physical activity questionnaire among older women," European Journal of Epidemiology, vol. 23, no. 10, pp. 661-667, 2008.

[22] H. Besson, S. Brage, R. W. Jakes, U. Ekelund, and N. J. Wareham, "Estimating physical activity energy expenditure, sedentary time, and physical activity intensity by self-report in adults," American Journal of Clinical Nutrition, vol. 91, no. 1, pp. 106-114, 2010. 


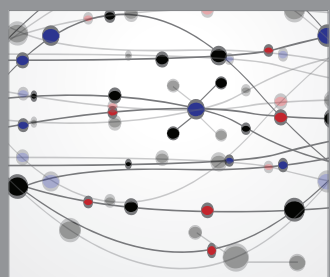

The Scientific World Journal
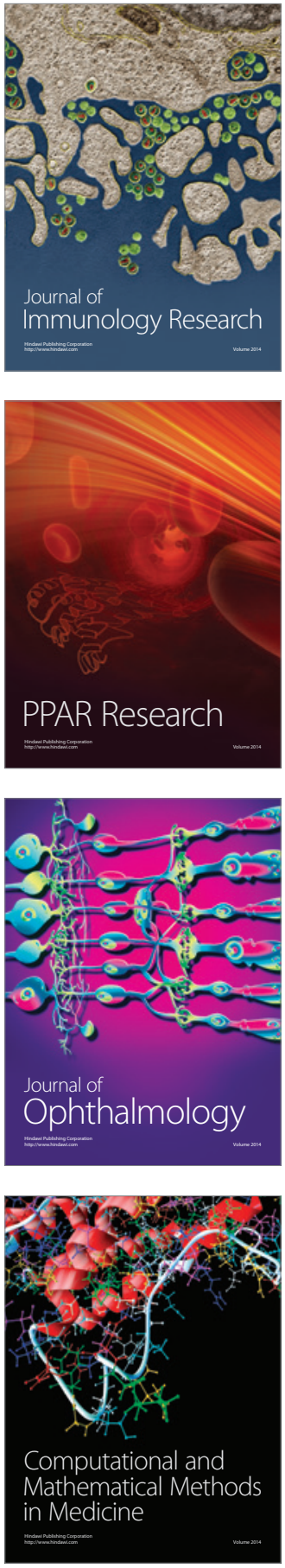

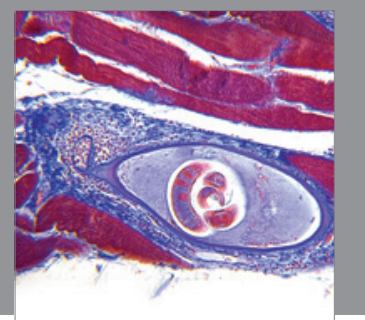

Gastroenterology

Research and Practice
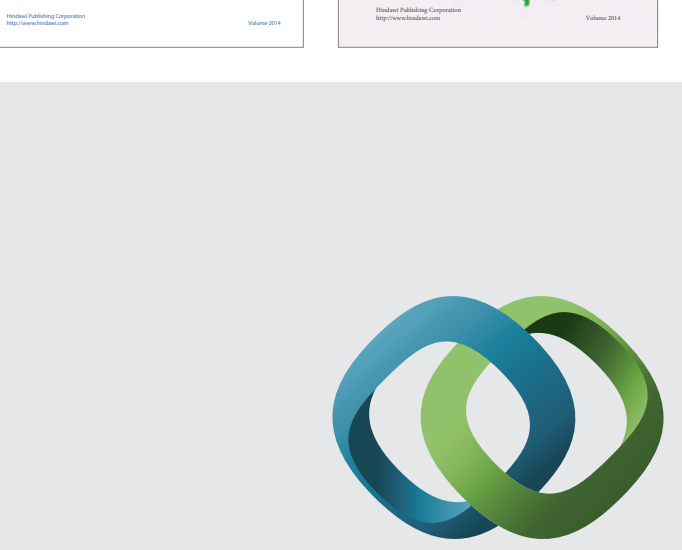

\section{Hindawi}

Submit your manuscripts at

http://www.hindawi.com
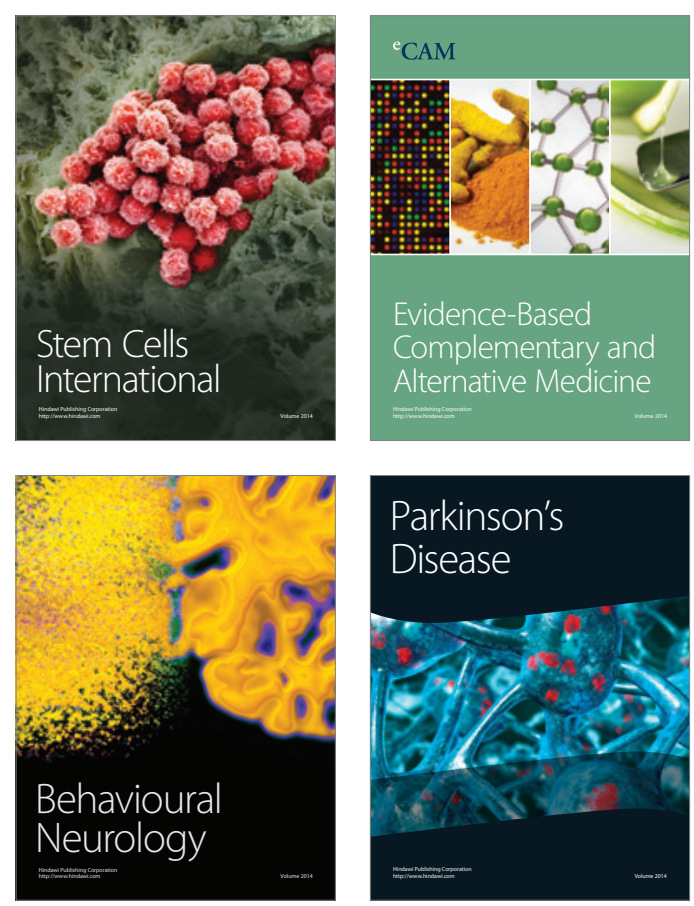

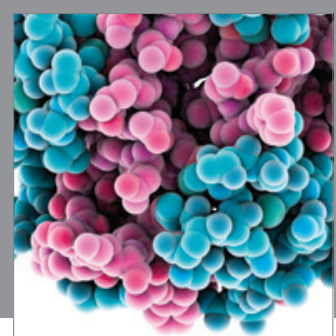

Journal of
Diabetes Research

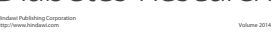

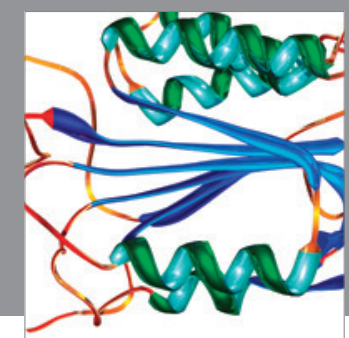

Disease Markers
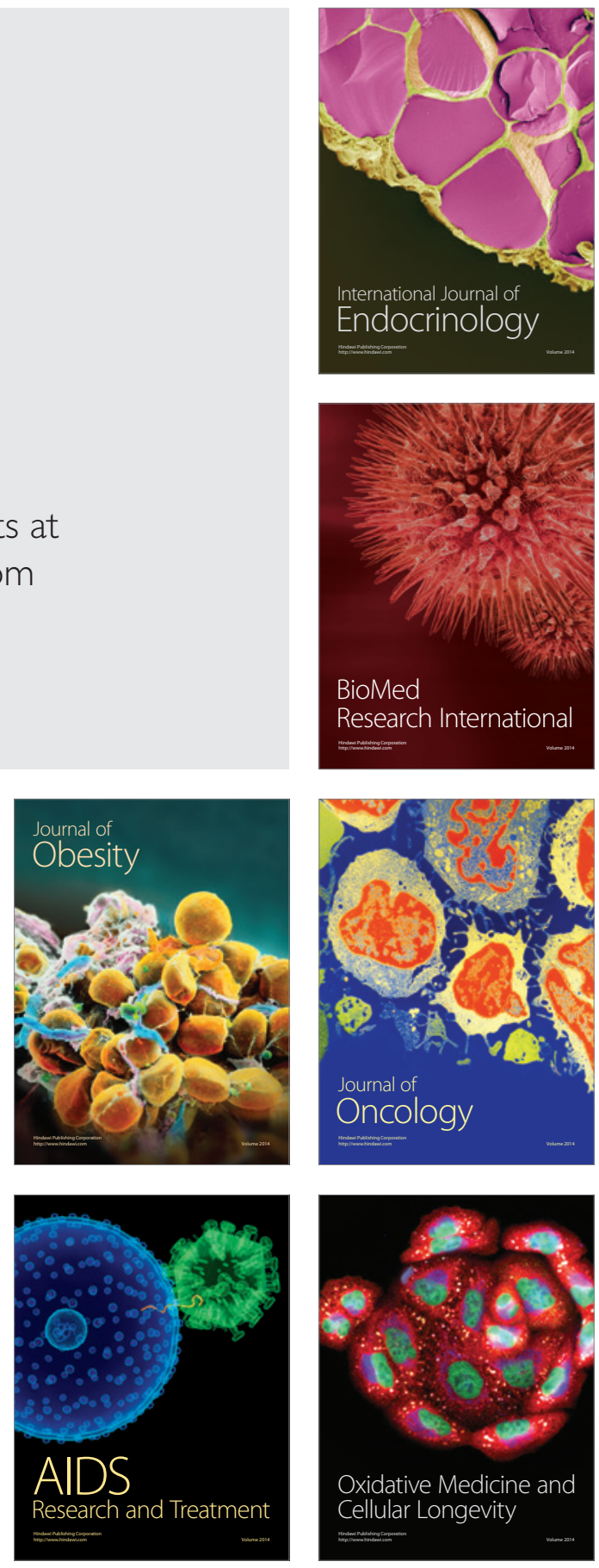